\title{
Lentivirus-TAZ Administration Alleviates Osteoporotic Phenotypes in the Femoral Neck of Ovariectomized Rats
}

\author{
Yan Zhang ${ }^{\mathrm{a}}$ Zheyang Wang ${ }^{\mathrm{b}}$ Lin Ding $^{\mathrm{c}}$ Ainiwaerjiang Damaolar ${ }^{\mathrm{d}}$ Zhiqiang Lie \\ Yusheng Qiue Zhanhai Yine \\ ${ }^{a}$ Center for Translational Medicine, The First Affiliated Hospital of Xi'an Jiaotong University, Xi'an, \\ 'Department of Orthopaedics, Ningbo First Hospital, Ningbo Hospital of Zhejiang University, Ningbo, \\ 'Department of Orthopaedics, The Rizhao Hospital of traditional Chinese medicine, Rizhao, \\ 'Department of Orthopaedics, Xinjiang Uyghur Municipal People's Hospital, Urumqi, e'Department of \\ Orthopaedics, The First Affiliated Hospital of Xi'an Jiaotong University, Xi'an, China
}

\section{Key Words}

Osteoporosis $\cdot$ TAZ $•$ Ovariectomized rat

\begin{abstract}
Background: Osteoporosis is characterized by impairment of bone mass, strength, and microarchitecture, leading to the susceptibility to fragility fractures, especially in femoral neck region. Transcriptional coactivator with PDZ-binding motif (TAZ) facilitates osteogenesis while suppressing adipogenesis via regulation of transcriptional activities of runt-related transcription factor 2 and peroxisome proliferator-activated receptor $\gamma$. Here, we validated the role of TAZ in vivo using an ovariectomized (OVX) rat model of osteoporosis. Methods: Serum alkaline phosphatase, triglyceride, cholesterol and urinary hydroxyproline were measured on an automatic analyzer using diagnostic reagent kits. Serum OCN and C-terminal crosslinked telopeptides of type I collagen were measured using ELISA. Bone mineral density was measured using dual-energy X-ray scanner. Mechanical parameters were detected by threepoint bending assays. Bone volume per tissue volume (BV/TV), trabecular thickness (Tb. Th), trabecular number (Tb. No), and trabecular separation (Tb. Sp) were measured by MicroCT. The mRNA and protein levels were quantified by Realtime PCR and Western Blotting respectively. Results: After injections of lentivirus overexpressing TAZ into the femoral neck region, bone mineral density, ultimate force, stiffness, BV/TV, Tb. Th, and Tb. No were significantly increased, whereas Tb. Sp was dramatically decreased. In the TAZ-overexpression region in the femoral neck of OVX rats, the mRNA levels of Runx2 and osteocalcin were obviously elevated, whereas that of PPARY and adipocyte protein 2 were downregulated. Conclusion: Lentivirus-mediated TAZ gene therapy alleviated the osteoporotic phenotypes in the femoral neck of OVX rats, providing an alternative strategy for the treatment of postmenopausal osteoporosis and prevention of osteoporotic fracture.
\end{abstract}

Y. Zhang, Z. Wang and L. Ding contributed equally to this work. 


\begin{tabular}{|c|c|}
\hline Cellular Physiology & Cell Physiol Biochem 2016;38:283-294 \\
\hline and Biochemistry & \begin{tabular}{l|l} 
DOI: 10.1159/000433629 \\
Published online: January 25, 2016
\end{tabular} $\begin{array}{l}\text { O 2016 Karger AG, Basel } \\
\text { www.karger.com/cpb }\end{array}$ \\
\hline
\end{tabular}

\section{Introduction}

Osteoporosis (OP) is a systemic metabolic skeletal disease, characterized by reduced bone mass and microarchitectural deterioration of bone tissue, leading to increased bone fragility and susceptibility to fractures [1]. According to data released by the World Health Organization (WHO), osteoporosis affects approximately 75 million people worldwide [2]. The incidence of osteoporosis commonly occurs in postmenopausal women due to estrogen deficiency, which is known as a critical factor impairing cancellous metaphyseal bone and reducing bone mineral density (BMD) [3]. Estrogen deficiency results in a marked bone loss due to imbalance between osteoclastic bone resorption and osteoblastic bone formation [4]. Despite major advances in current anti-osteoporotic agents suppressing osteoclastic bone resorption (e.g., bisphosphonates) or stimulating osteoblastic bone formation (e.g., the growth factors, hormones) [5], the side effect of these agents could not be ignored [6]. Therefore, there is an urgent need to find new drugs for prevention and treatment of osteoporosis-associated fracture.

The fundamental cause of postmenopausal osteoporosis is enhanced osteoclastic bone resorption and impaired osteoblastic bone formation [7]. Bone formation is mediated by osteoblast, which is derived from mesenchymal stem cells (MSCs) [8]. MSCs are pluripotent cell type that can differentiate into several distinct lineages, including osteoblasts driven by runt-related transcription factor 2 (Runx2), and adipocytes driven by peroxisome proliferator-activated receptor $\gamma$ (PPAR $\gamma$ ) [9]. Runx2 and PPAR $\gamma$ are under a delicate regulation to specify the alternate cell fates of MSCs [10]. Transcriptional coactivator with PDZ-binding motif (TAZ) is involved in this delicate regulation by co-activating Runx2dependent gene transcription while simultaneous repressing PPAR $\gamma$-dependent gene transcription [11]. These effects occur through direct interaction between TAZ and Runx2 or PPAR $\gamma$. TAZ contains a 14-3-3 binding motif, a WW domain, a transcriptional activation domain, and a C-terminal motif that can interact with PDZ domain-containing proteins [12, 13]. The WW domain of TAZ binds strongly to the PPxY motif (Pro-Pro-X-Tyr) in Runx2 and transcriptionally activates Runx2-driven genes [14]. In contrast to the stimulatory effect, TAZ binds to but markedly inhibits the ability of PPAR $\gamma$ to drive the expression of fat cell genes such as adipocyte protein 2 (aP2), and depletion of TAZ in MSCs dramatically increases their adipogenic potential [15]. Therefore, TAZ functions as a molecular rheostat that modulates MSCs differentiation and stimulates bone development while simultaneously blocking the differentiation of MSCs into fat.

In the present study, we examined the effect of lentivirus-mediated TAZ gene transduction on the phenotypes of an ovariectomized (OVX) rat model of osteoporosis. We found that the bone mineral density (BMD), ultimate force, stiffness, bone volume per tissue volume (BV/ $\mathrm{TV}$ ), trabecular thickness (Tb.Th), trabecular number (Tb.No) were significantly increased after TAZ was overexpressed, whereas trabecular separation (Tb.Sp) was dramatically decreased. Our results demonstrated that TAZ gene therapy alleviated the osteoporotic phenotypes in the femoral neck region of ovariectomized rats, providing a novel strategy for prevention and treatment of postmenopausal osteoporosis and osteoporosis-associated fracture.

\section{Materials and Methods}

\section{Animal and Treatments}

Six-month old female Sprague-Dawley (SD) rats were housed in an air-conditioned room at $23 \pm 1^{\circ} \mathrm{C}$, $55 \%-60 \%$ relative humidity, with a $12 \mathrm{~h}$ light/dark cycle. Rats were anesthetized with $10 \%$ of chloral hydrate and ovaries were removed bilaterally. A sham operation, during which the ovaries were just touched with forceps, was performed in the sham group. Three months after surgery, rats were divided into 4 treatment groups ( $(n=6)$ : (1) OVX (no treatment); (2) OVX-drill; (3) OVX-LentiCherry (lentivirus expressing Cherry); (4) OVX-LentiTAZ (lentivirus expressing TAZ). Lentivirus $\left(2 \times 10^{9} \mathrm{UT} / \mathrm{ml}\right)$ was mixed with AteloGene 


\section{Cellular Physiology Cell Physiol Biochem 2016;38:283-294

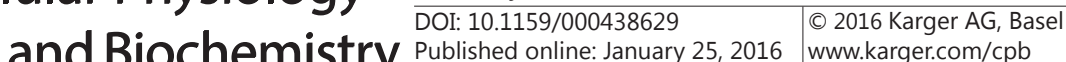 \\ Published online: January 25, 2016 Ww.kargem/cpb}

(CosmoBio, Japan) at ratio of 1:1, and $5 \mu \mathrm{l}$ of lentivirus mixture were injected into the femoral neck of the rats. All groups were observed for three months. At the indicated time points, body weight and femoral neck BMD of the rats were examined. Blood was collected via the vena ophthalmica, urinary and serum parameters were determined using ELISA. The femoral necks of the rats were dissected for biomechanical parameters determination and staining analysis. All animal experiments were carried out according to the guidelines of the Xi'an Jiaotong University Animal Care and Use Committee.

\section{Biochemical Analysis of Serum and Urine Specimens}

The blood and urine of the rats were collected. The levels of serum alkaline phosphatase (ALP), triglyceride (TG), cholesterol (CHO) and urinary hydroxyproline (HOP) concentrations were measured on an automatic analyzer (Ciba-Corning 550, USA) using a diagnostic reagent kit. The levels of serum OCN concentrations were measured using commercially available kits (Shanghai Haling biological technology, PR China). Serum levels of C-terminal cross-linked telopeptides of type I collagen (CTX) were measured using ELISA kit (Immunodiagnostic Systems, Boldon, UK).

\section{Bone Mineral Density Measurements}

The BMD of the right femoral neck was measured using dual-energy X-ray absorptiometry scanner (DEXA, GE Healthcare, USA) for bone density assessment in small laboratory animals mode. Calibration of the instrument was conducted as recommended by the manufacturer. Quality control with BMD $(0.0553 \mathrm{~g} /$ $\mathrm{cm}^{2}$ ) and percentage fat composition $(16.7 \%)$ of the phantom were performed each time the instrument was switched on. The measurements were expressed as grams of mineral contents per $\mathrm{cm}^{2}$ of surface area. All rats were placed in the same direction.

\section{Mechanical Testing Experiment}

The left femurs were slowly thawed at room temperature. The femurs were submitted to a threepoint bending test using a material testing machine (MTS 858 Mini Bionix II, MTS Systems Corp., USA). Each femur was placed in the machine with two support points and loaded at a speed of $2 \mathrm{~mm} / \mathrm{min}$. In the movement of the central loading point, the load and displacement were recorded until the specimen was broken. Using the load deformation curve, biomechanical parameters including the ultimate force and stiffness were calculated.

\section{MicroCT Analysis}

The right femur was fixed in 10\% ethanol and was scanned at 8-mm resolution using a Scancom CT-35 instrument (Scanco Medical). Bone parameters including BV/TV, Tb. Th, Tb. No, and Tb. Sp were calculated using Scanco software to analyze the trabecular region of the femoral neck.

\section{Immunohistochemistry Analysis}

After MicroCT scanning, the $4 \mu \mathrm{m}$-sections of femoral neck were prepared, and was stained using hematoxylin and eosin (H\&E). The expression of TAZ was evaluated using immunohistochemical analysis. Briefly, the endogenous peroxidase activity was blocked using $3 \% \mathrm{H}_{2} \mathrm{O}_{2}$ for $1 \mathrm{~h}$, followed by incubation with 5\% Serum-Free Protein Block and then primary antibody anti-TAZ (1:100) at $4{ }^{\circ} \mathrm{C}$ overnight. Immunodetection was performed in a 3-step protocol, using streptavidin-horseradish peroxidase complex, with visualization by 3,3-diaminobenzidine.

\section{Realtime PCR}

Total RNA was obtained from the femoral neck of the rats using Trizol (Invitrogen). Quantitative realtime PCR was performed in BioRad CFX96 real-time PCR system, using SYBR Premix EX Taq (TaKaRa). Gene expression was quantified by the comparative $2^{-\Delta \Delta \mathrm{ct}}$ method using the GAPDH gene as normalization control. Melting curves were used to check for PCR specificity. The primer sequences were as follows: TAZ: $5^{\prime}$-agg atc agg atg cgt caag-3' and 5'-cca aag tcc cga ggt caa-3'; GAPDH: 5'-gaa agc tgt ggc gtg atgg-3' and 5'-gta ggc cat gag gtc cacca-3'; Runx2: 5'-cag gtt caa cga tct gag att tgt-3' and 5'-tga aga ccg tta tgg tca aag tga-3'; OCN: 5'-agg acc ctc tct ctg ctcac-3' and 5'-gct cac aca cct ccct-3'; PPAR $\gamma: 5^{\prime}$-ctg tga agt tca atg cac tgg aat-3' and 5'-atg ggc ttc acg ttc agcaa-3'; aP2: 5'-tcc ttc aaa ctg ggc gtg gaa-3' and 5'-cca ggg tta tga tgc tct tcact-3'.

\section{KARGER}




\section{Cellular Physiology Cell Physiol Biochem 2016;38:283-294 \\ and Biochemistry \begin{tabular}{l|l} 
Dublished online: January 25, 2016 & $\begin{array}{l}\text { (c) } 2016 \text { Karger AG, Basel } \\
\text { www.karger.com/cpb }\end{array}$
\end{tabular} \\ Zhang et al.: TAZ Alleviates Osteoporotic Phenotypes}

\section{Western Blotting}

Femurs were excised and all muscles and connective tissue was removed. The femoral neck were immediately frozen in liquid nitrogen and stored at $-80^{\circ} \mathrm{C}$ until used. Bone protein was extracted as previously described [16]. The protein was quantified and separated by sodium dodecyl sulfatepolyacrylamide gel electrophoresis (SDS-PAGE) and transferred onto a polyvinylidene difluoride (PVDF) membrane. After being blocked with $5 \%$ milk, the membranes were probed with specific antibodies overnight at $4{ }^{\circ} \mathrm{C}$, followed by incubation with the corresponding secondary antibodies at room temperature for $1 \mathrm{~h}$. The blots were visualized with ECL-plus reagent (AmershamPharmacia Biotech, USA). The mouse monoclonal antibody against TAZ (TA503538) was obtained from OriGene Technologies (Rockville, MD, USA).

\section{Statistical Analysis}

The data are expressed as mean \pm standard deviation. Statistical analysis was performed using oneway ANOVA combined with Bonferroni's multiple comparison test using SPSS 13.0. A $P$ value $<0.05$ was considered significant.

\section{Results}

Osteoporotic phenotypes induced by ovariectomy (OVX)

To examine the in vivo effects of TAZ gene transduction on estrogen deprivation-induced osteoporosis in rats, OVX was performed at the age of 6 months. 3, 4, 5, or 6 months after OVX was performed, serum and urine were collected for determination of bone formation indicators (ALP and OCN) and bone resorption indicators including serum C-terminal crosslinked telopeptides of type I collagen (CTX) and urinary hydroxyproline (HOP). As shown in Fig. 1A, the concentration of ALP were increased to $150 \mathrm{U} / \mathrm{l}$ approximately 3 months after OVX, and the extent of increase became bigger in month 4, 5, and 6. Similarly, the change of OCN after OVX was consistent with that of ALP (Fig. 1B). Bone resorption indicators including serum CTX and urinary HOP were quantified. As shown in Fig. 1C and D, serum CTX levels and urinary HOP levels were significantly elevated after OVX.

Then the femurs of the rats were collected at the indicated time points for determination of BMD, biomechanical parameters and histomorphology analysis. As shown in Fig. 1E, the BMD of femoral neck decreased significantly 3 months after OVX. Three-point bending test demonstrated that ultimate force and stiffness downregulated obviously after OVX (Fig. 1F and G). H\&E staining confirmed the microarchitectural of femoral necks after the rats were ovariectomized (Fig. 1H). Therese results collectively demonstrated that after OVX the rats displayed apparent osteosteoporotic phenotypes, and were suitable for validating the role of TAZ in vivo.

Lentivirus-TAZ administration mediated efficient gene delivery in the rat femoral necks

We packaged lentivirus overexpressing TAZ or Cherry (used as negative control) and injected the lentivirus into the femoral neck of ovariectomized rats. The flow plan of the procedures was shown in Fig. 2A. At the indicated time points after lentivirus was administrated, the RNA and protein were extracted from the femoral neck to confirm the efficiency of TAZ overexpression. As shown in Fig. 2B, the mRNA levels of TAZ in the femoral neck of OVX-TAZ rats were significantly higher than controls during the time window we detected. The protein levels of TAZ were consistent with the mRNA results (Fig. 2C). Immunohistochemistry results further confirmed the overexpression of TAZ in the femoral neck of OVX-TAZ rats (Fig. 2D) 3 months after OVX. Collectively, these results confirmed the efficiency of TAZ gene transduction mediated by lentivirus.

Local lentivirus-TAZ administration did not affect systemic biochemical indicators of bone formation and resorption

After the overexpression of TAZ was validated, we further detected the levels of biochemical indicators of bone formation and resorption. As shown in Fig. $3 \mathrm{~A}$ and $\mathrm{B}$, the 


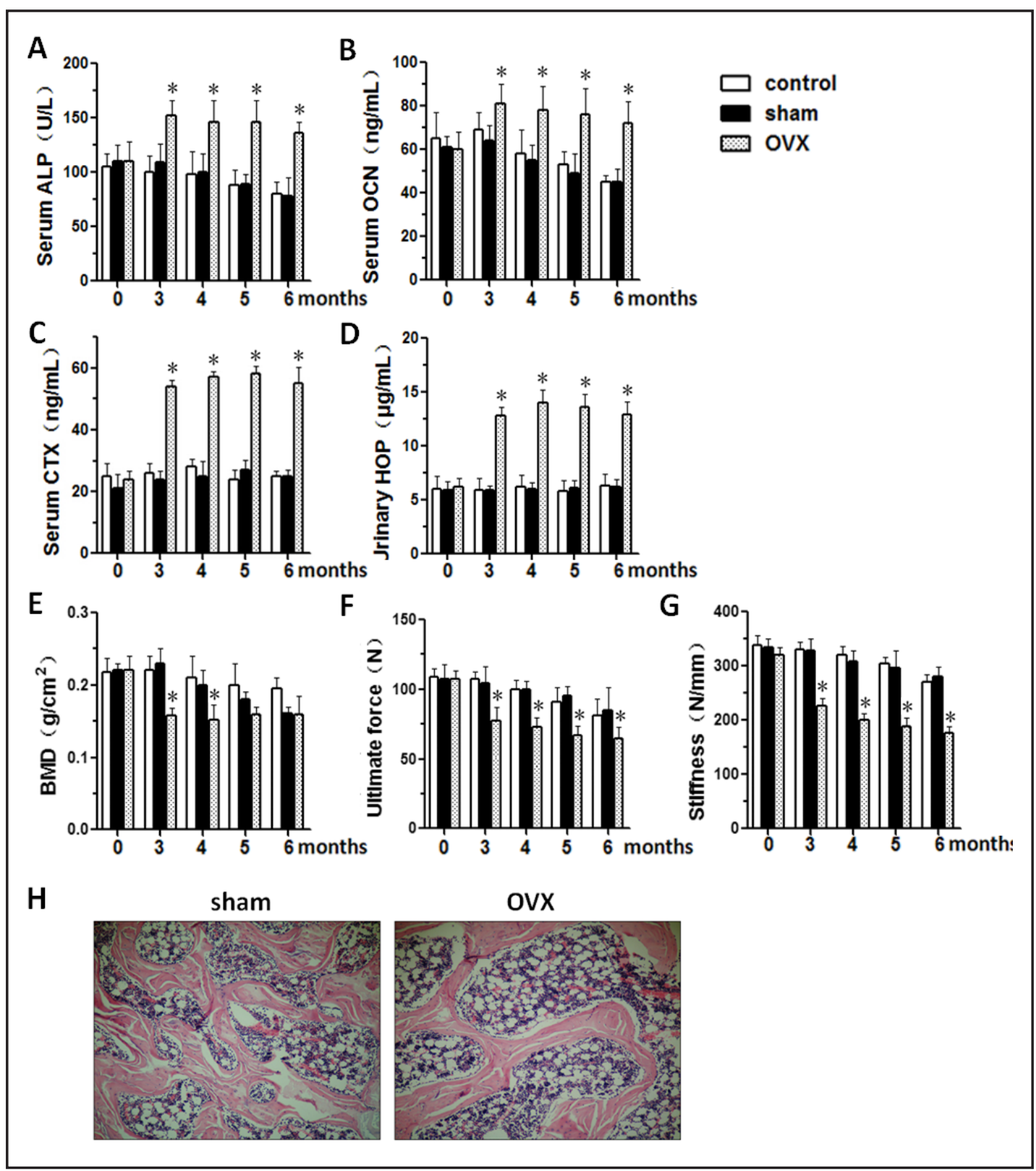

Fig. 1. Osteoporotic phenotypes induced by ovariectomy (OVX). (A) At the indicated time points after OVX was performed, serum of differently treated rats were collected for determination of ALP using an automatic analyzer. (B) The serum levels of OCN were determined by ELISA. (C) The serum levels of CTX were determined by ELISA. (D) At the indicated time points after OVX was performed, urine of differently treated rats were collected for determination of HOP using an automatic analyzer. (E) At the indicated time points after OVX, the BMD of the right femoral necks of differently treated rats were measured using DEXA. (F) The ultimate force of the left femoral necks was detected using a three-point bending test. (G) The stiffness of the left femoral necks was detected using a three-point bending test. $(\mathrm{H})$ The microarchitectural of the femoral neck trabecular was determined by H\&E staining.

concentration of ALP and OCN were not changed when TAZ was introduced into the rat femurs. Additionally, the levels of bone resorption indicators, including serum CTX and urinary HOP, also displayed a similar pattern after TAZ was overexpressed (Fig. 3C and D). These results demonstrated that local TAZ administration did not induced the levels of bone metabolism indicators in ovariectomized rats. 
A

Ovarietomy/sham Drill/drill+lentivirus injection

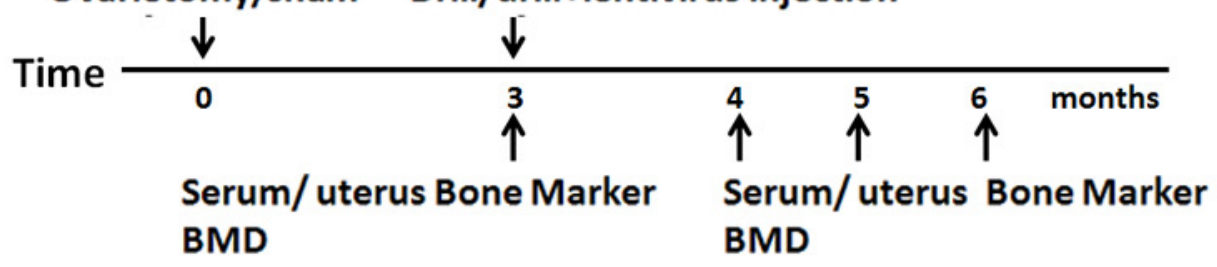

B

\section{Biomechanical properties}

$\stackrel{N}{\leftarrow}$ Histomorphometry

\section{Biomechanical properties} Histomorphometry

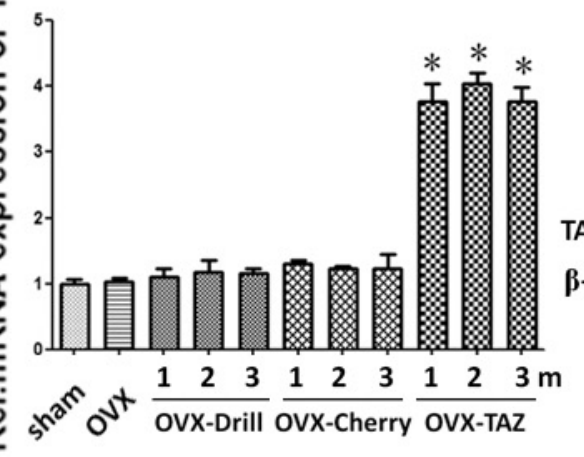

C

D
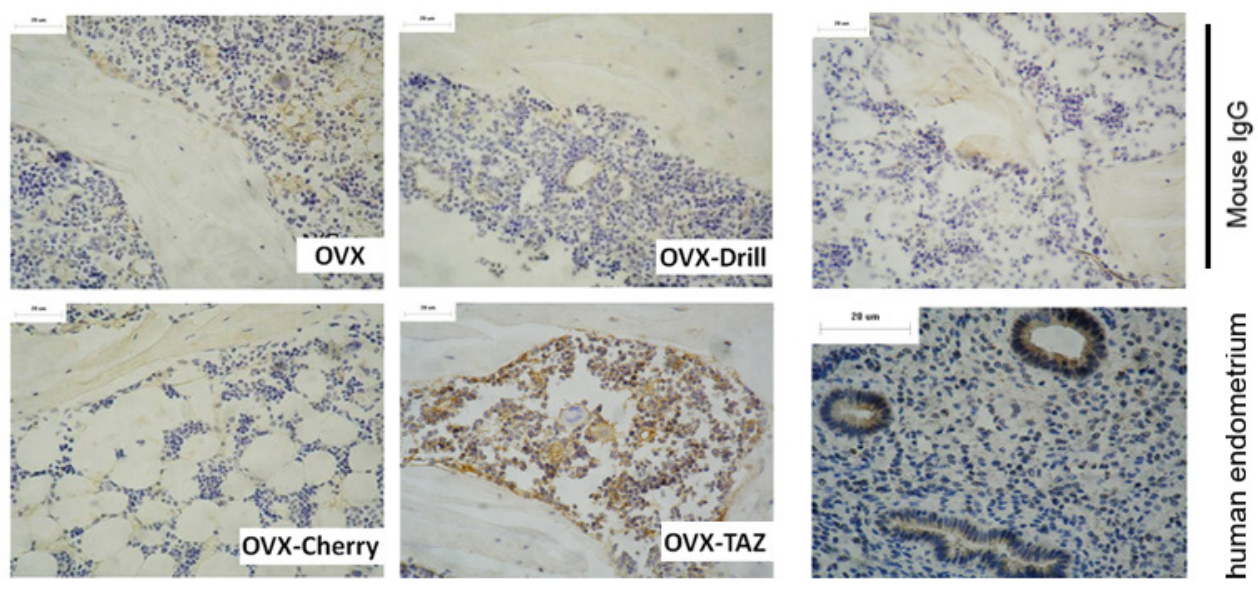

anti-TAZ
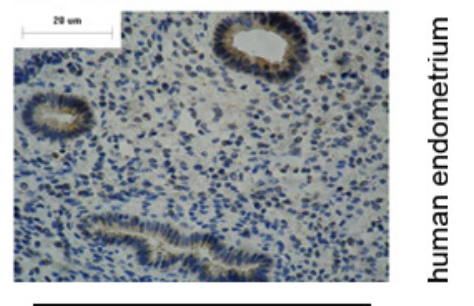

anti-TAZ

Fig. 2. The efficiency of lentivirus-TAZ mediated gene delivery in the rat femoral necks. (A) The flow plan of the experiment procedures. (B) At the indicated time points after lentivirus was administrated, total RNA of the rat femoral necks were extracted, and relative mRNA levels of TAZ were determined by Realtime PCR. (C) The protein level of TAZ of the rat femoral necks was determined by Western blotting. (D)Three months after lentivirus-TAZ was administrated, the expression of TAZ was confirmed by immunohistochemistry analysis. Mouse IgG for OVX-TAZ group was used as negative control and anti-TAZ antibody for human endometrium tissue was used as positive control.

Lentivirus-TAZ administration increased BMD and mechanical capability of the femurs of oVX-rats

Then we collected the femurs of OVX-rats at the indicated time point, examined the BMD using DEXA, and detected the ultimate force and stiffness by performing three-point bending test. As shown in Fig. 4A, the value of BMD of femoral neck increased approximately by 2 -fold when TAZ was introduced into the femoral necks of OVX-rats, and this increase 


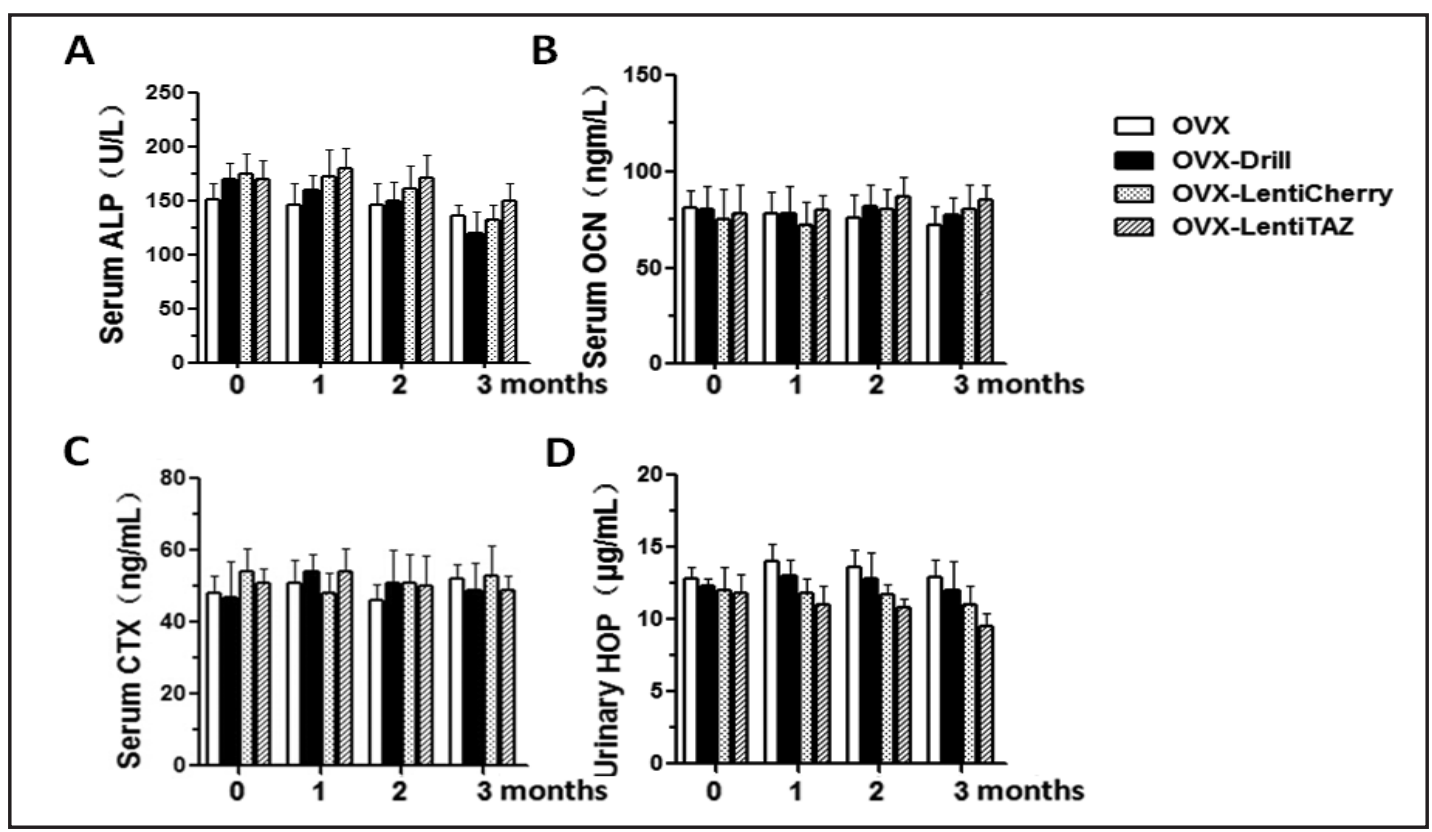

Fig. 3. The biochemical indicators of bone formation and resorption. (A) At the indicated time points after lentivirus was administrated, serum of differently treated rats were collected for determination of ALP using an automatic analyzer. (B) The serum levels of OCN were determined by ELISA. (C) The serum levels of CTX were determined by ELISA. (D) The urinary levels of HOP were measured using an automatic analyzer.

Fig. 4. The BMD and mechanical capability of the femurs. (A) At the indicated time points after lentivirus was administrated, the BMD of the right femoral necks of differently treated rats were measured using DEXA. (B) The ultimate force of the left femoral necks was detected using a three-point bending test. (C) The stiffness of the left femoral necks was detected using a three-point bending test.

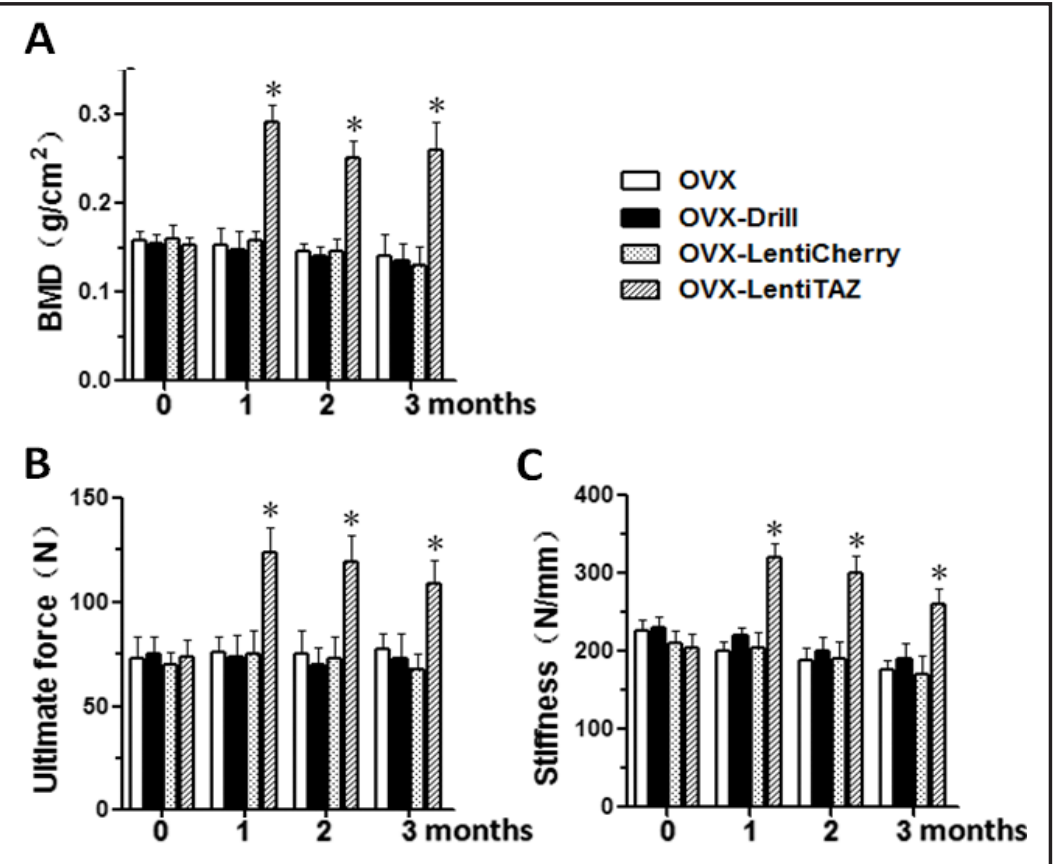

lasted for at least 3 months after lentivirus infection. Similarly, the ultimate force and stiffness increased significantly after TAZ was overexpressed (Fig. 4B and C). These results demonstrated that TAZ overexpression improved the quantity and quality of rat femurs.

The effect of lentivirus-TAZ administration on femoral neck histomorphology

The femurs of OVX-rats were analyzed by MicroCT. Bone parameters including BV/TV, $\mathrm{Tb}$. Th, Tb. No, and Tb. Sp were calculated using Scanco software to analyze the trabecular 


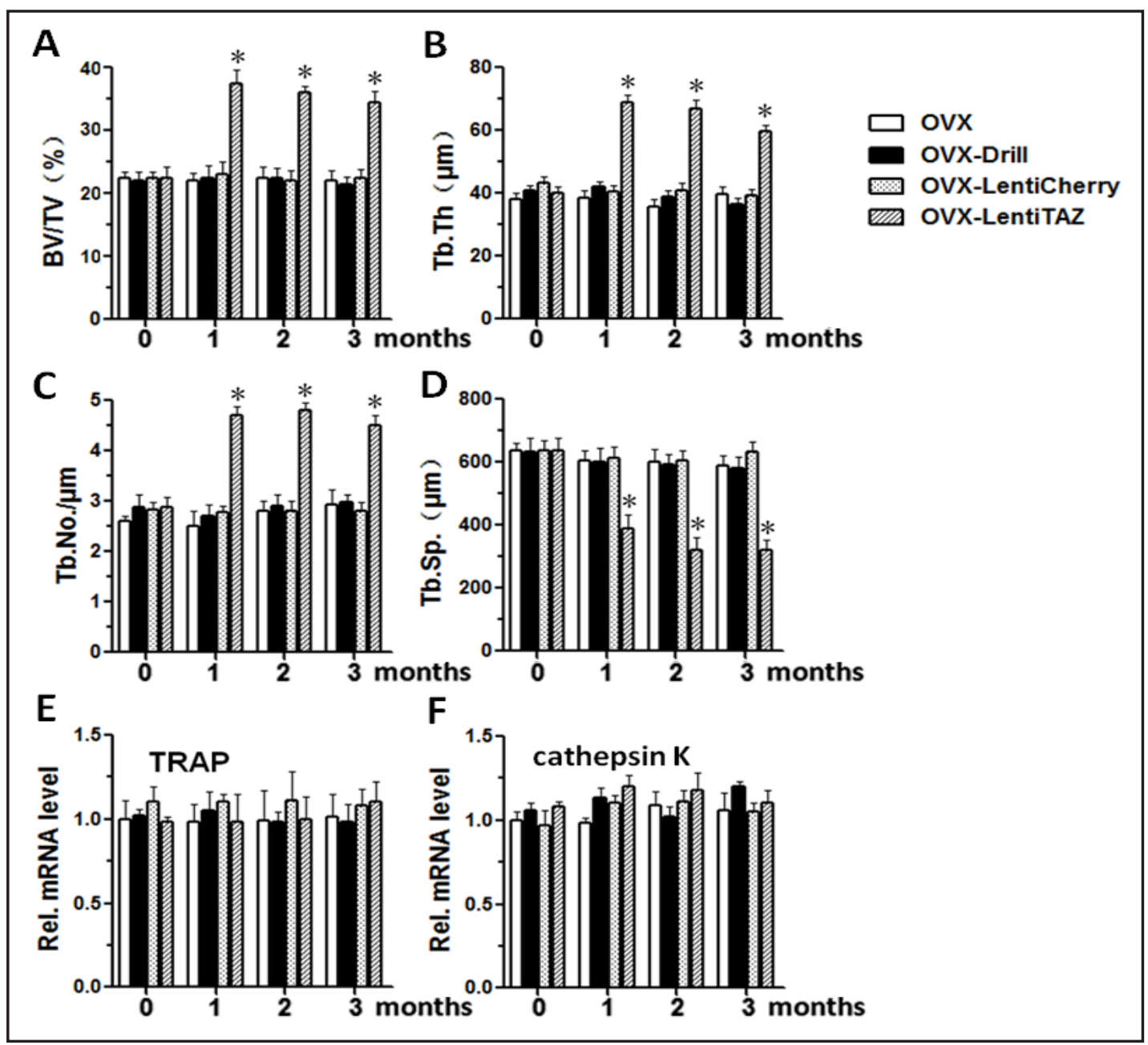

Fig. 5. The effect of lentivirus-TAZ administration on the histomorphology of femoral neck. (A) At the indicated time points after lentivirus was administrated, the BV/TV of the right femoral necks of differently treated rats was analyzed using MicroCT scanning. (B) The Tb. Th of the right femoral necks was analyzed using MicroCT scanning. (C) The Tb. No of the right femoral necks was analyzed using MicroCT scanning. (D) The Tb. Sp of the right femoral necks was analyzed using MicroCT scanning. (E) At the indicated time points after lentivirus was administrated, total RNA of the left femoral necks of differently treated rats were extracted, and relative mRNA levels of TRAP were determined by Realtime PCR. (F) Relative mRNA levels of cathepsin K were determined by Realtime PCR.

region of the femoral neck. As shown in Fig. 5A, the ratio of bone volume to total tissue volume (BV/TV) increased significantly and lasted at least for 3 months when TAZ was introduced into the OVX-rats via lentivirus. The thickness of trabecular (Tb.Th) was increased dramatically after TAZ was overexpressed (Fig. 5B). Similar changes also occurred in the number of trabecular (Tb. No, Fig. 5C). Consistently, the separation extent of trabecular (Tb. Sp) was downregulated after TAZ was overexpressed locally (Fig. 5D). To exclude the possibility that increase of bone mass is caused by decreased bone resorption induced by TAZ, we detected the mRNA expression of osteoclastic specific genes, including tartrateresistant acid phosphatase (TRAP) and Cathepsin K. The mRNA levels of TRAP (Fig. 5E) and Cathepsin K (Fig. 5F) were not changed after TAZ administration, demonstrating that TAZ did not affect bone resorption. These results further confirmed the function of TAZ in stimulating osteoblastogenesis and bone formation. 
Fig. 6. The effect of lentivirus-TAZ administration on the lipometabolic markers. (A) At the indicated time points after lentivirus was administrated, total RNA of the left femoral necks of differently treated rats were extracted, and relative mRNA levels of Runx2 were determined by Realtime PCR. (B) Relative mRNA levels of OCN were determined by Realtime PCR. (C) Relative mRNA levels of PPARY. (D) Relative mRNA levels of aP2. (E) The bodyweights of the rats at the indicated time points after lentivirus was administrated. (F) At the indicated time points after lentivirus was administrated, serum of differently treated rats were collected for determination of TG using an automatic analyzer. (G) The serum levels of Cho were matic analyzer. determined using an auto-
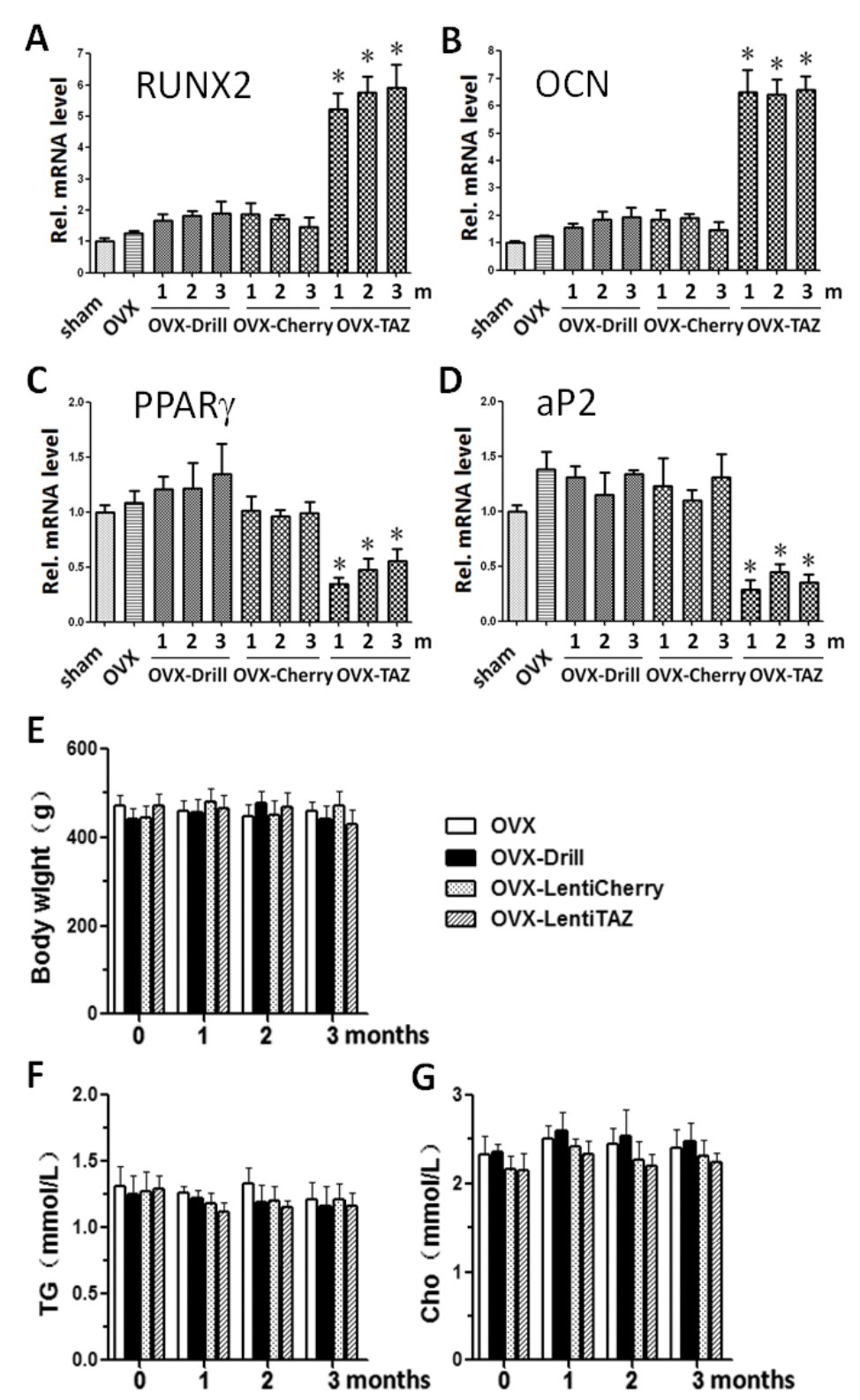

The effect of lentivirus-TAZ administration on the lipometabolic markers

Given that TAZ functions as a switch in MSCs differentiation and stimulates osteoblastogenesis while repressing adiocytogenesis, we detected the mRNA levels of osteoblastic and adipogenic markers in the femoral neck. As expected, when TAZ was overexpressed the mRNA levels of Runx2 were significantly induced (Fig. 6A). The mRNA levels of OCN also showed similar changes (Fig. 6B). However, the mRNA levels of adipogenic markers, PPAR $\gamma$ and aP2 were dramatically suppressed (Fig. 6C and D).

After confirming the effect of TAZ in regulation of PPAR $\gamma$ and $\mathrm{aP} 2$, we detected the serum lipometabolic indicators, including triglyceride (TG) and cholestrol (CHO). As demonstrated in Fig. 6E, the body weights were not affected by TAZ gene delivery. Furthermore, the levels of TG remained unchanged when TAZ was overexpressed (Fig. 6F). Another lipometabolic indicator, $\mathrm{CHO}$ also displayed a similar trend (Fig. 6G). These results collectively demonstrated that TAZ gene delivery suppressing local adicytogenesis in vivo. 


\section{Cellular Physiology Cell Physiol Biochem 2016;38:283-294 \\ \begin{tabular}{l|l|l} 
DOI: $10.1159 / 000438629$ & 2016 Karger AG, Basel
\end{tabular} \\ Zhang et al.: TAZ Alleviates Osteoporotic Phenotypes}

\section{Discussion}

Osteoporosis is a condition characterized by a decrease in the density of bone, decreasing its strength and resulting in fragile bones [1]. Osteoporosis affects 55\% of Americans aged 50 and above. Of these, approximately $80 \%$ are postmenopausal women [17]. Postmenopausal osteoporosis is attributable to the estrogen deficiency after menopause. Estrogen deficiency increases bone resorption and influences bone turnover and bone loss [3]. Clinically, hormone replacement therapy (HRT) has been widely used as a therapeutic strategy for postmenopausal osteoporosis [18]. However, long-term application of HRT has potential malignant effects on reproductive systems [19]. Other drugs that stimulate bone formation (e.g., growth hormone, and parathyroid hormone) or inhibit bone resorption (e.g., bisphosphonates) may prevent bone loss progression, but with compromised efficacy due to the coupling regulation between bone resorption and formation $[5,6]$. Osteoporotic bones, such as distal radius, vertebral body and femoral neck, are vulnerable to minor injuries, which will result in osteoporosis-associated fractures. Osteoporotic fractures impair a patient's function and quality of life and represent one of the major public health burdens. Demographic changes predict a dramatic increase in osteoporosis-associated fractures. Therefore there is a high demand to find an efficient and less harmful way to prevent and treat osteoporosis and osteoporosis-associated fractures.

The increase in marrow adipogenesis associated with osteoporosis and age-related osteopenia is well known clinically. However, we are only now beginning to understand the mechanisms that control the differentiation of MSCs to either osteoblasts or adipocytes.TAZ has been linked with the development of bone, fat, limb, kidney, muscle, heart and lung tissues, and functions as a molecular rheostat, promoting MSCs differentiation into osteoblastic lineages by coactivating Runx 2 and blocking MSCs differentiation into adipocyte lineages by repressing PPAR $\gamma$-dependent gene transcription [11-15]. Promoting MSCs differentiate into osteoblasts other than adipocytes represents a promising direction for the targeted strategy for the prevention and treatment of postmenopausal osteoporosis. Therefore, TAZ could be exploited as a potential target.

In this study, we used ovariectomized rat model to systematically investigate the effects of local TAZ gene delivery on prevention and treatment of estrogen deficiency-induced osteoporosis. The ovariectomized rat model is a widely used model to evaluate the efficacy of new drug entities to prevent or treat postmenopausal osteoporosis. Surgical ovariectomy results in a state of high bone turnover and rapid bone loss, which have many similarities between the phenotypes observed in OVX rats and humans [20]. Here, we found that ovariectomy significantly increased serum ALP and OCN, as well as serum CTX and urinary HOP compared with the sham rats. Moreover, ovariectomy reduced BMD, biomechanical characters (ultimate force and stiffness), and deteriorated the microarchitecture of trabecular. Injections of lentivirus overexpressing TAZ into the femoral neck of OVX rats significantly increased the BMD, ultimate force, and stiffness of the injected region. Lentivirus-TAZ administration decreased Tb. Sp in OVX rats and significantly increased BV/TV, Tb. Th, and $\mathrm{Tb}$. No. in the same region. Based on the effect of lentivirus-TAZ on the phenotypes of femoral neck region in OVX rats, our results confirmed that TAZ has potential to be utilized as an alternative target for the prevention and treatment of postmenopausal osteoporosis and osteoporosis-associated fracture.

Serum levels of ALP and OCN are phenotypic markers of osteoblastic differentiation and bone formation, while serum CTX and urinary HOP serve similar roles in monitoring bone resorption [21]. After OVX, the serum levels of ALP and OCN were significantly increased, indicating an increased bone formation. This is consistent with the previous reports that estrogen deficiency-induced osteoporosis is high turnover type of osteoporosis [4]. We did not detect the alteration of serum bone resorption indicators. TAZ gene administration in the femoral neck of ovariectomized rats did not change the serum levels of bone formation indicators, either or that of adipogenesis indicators, that is probably because local gene delivery could alleviate the local osteoporotic phenotypes but not that of whole body. 


\section{Cellular Physiology Cell Physiol Biochem 2016;38:283-294 \begin{tabular}{l|l|l|} 
DOI: 10.1159/000438629 2016 Karger AG, Basel & (c) \\
and Biochemistry Published online: January 25, 2016 & wwwkargercom/cpb
\end{tabular} \\ Zhang et al.: TAZ Alleviates Osteoporotic Phenotypes}

BMD is the gold standard for the evaluation of osteoporosis risk [22]. In our study, BMD of femoral neck was markedly decreased in the OVX rats compared with the sham controls. The improved BMD during the 3-months treatment with lentivirus-TAZ indicated that TAZ could prevent the bone loss induced by ovariectomies. Both BMD and trabecular bone microarchitecture may improve bone strength. Bone microarchitecture and remodeling are assessed by MicroCT analysis, which is a widely used method to analyze bone volume and architecture [23]. In the present study, we analyzed BV/TV, Tb. Th, Tb. N and Tb. Sp of the femoral necks as the structural parameters. Our results revealed that lentivirus-TAZ treatment obviously increased BV/TV, Tb. No, Tb. Th and decreased Tb. Sp compared to the OVX group. These histomorphological parameters strongly supported TAZ as an inhibitor of OVX-induced bone loss.

Lentivirus-mediated gene therapy is a promising strategy for the prevention and treatment of many diseases. Lentivirus can infect both dividing and non-dividing cells. After enter the cell, the genes that lentivirus carries could integrate into the genome of the host cell, and express with a sustained manner. Moreover, lentivirus could be easily packaged and purified at a high titer [24]. Therefore, it is of great value to exploit lentivirus as vector for gene therapy.

In summary, this is an original report to demonstrate that the exogenous delivery of TAZ can improve the quantity and quality of bone in an OVX-rat model of osteoporosis. This study may enable the development of a potential therapeutic option for the relief of estrogen deficiency-induced osteoporosis, and prevention and treatment of osteoporosis-associated fracture in postmenopausal women.

\section{Acknowledgments}

This work is supported by the Fundamental Research Funds for the Central Universities of China, National Natural Science Foundation of China (No. 30801173, 81371943, 81300716) and Shaanxi Provincial Program for Science and Technology Development(2015SF136).

\section{Disclosure Statement}

The authors state that they have no conflicts of interest.

\section{References}

1 Urano T, Inoue S: Genetics of osteoporosis. Biochem Biophys Res Commun 2014;452:287-293.

2 Wade SW, Strader C, Fitzpatrick LA, Anthony MS, O'Malley CD: Estimating prevalence of osteoporosis: examples from industrialized countries. Arch Osteoporos 2014;9:182.

3 Rao SK, Rao AP: A literature review and case series of accelerating fracture healing in postmenopausal osteoporotic working women. J Orthop 2014;11:150-152.

4 Manolagas SC, O'Brien CA, Almeida M: The role of estrogen and androgen receptors in bone health and disease. Nat Rev Endocrinol 2013;9:699-712.

5 Bernabei R, Martone AM, Ortolani E, Landi F, Marzetti E: Screening, diagnosis and treatment of osteoporosis: a brief review. Clin Cases Miner Bone Metab 2014;11:201-207.

6 Tandon VR, Sharma S, Mahajan A: Bisphosphonate drug holidays: Can we recommend currently? J Midlife Health 2014;5:111-114.

7 McLendon AN, Woodis CB: A review of osteoporosis management in younger premenopausal women. Womens Health (Lond Engl) 2014;10:59-77.

8 Knight MN, Hankenson KD: Mesenchymal Stem Cells in Bone Regeneration. Adv Wound Care (New Rochelle) 2013;2:306-316. 


\section{Cellular Physiology Cell Physiol Biochem 2016;38:283-294

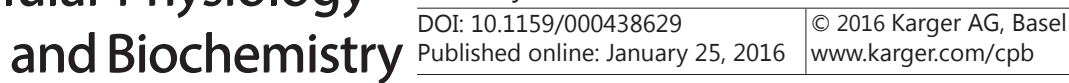 \\ Zhang et al.: TAZ Alleviates Osteoporotic Phenotypes}

9 James AW: Review of Signaling Pathways Governing MSC Osteogenic and Adipogenic Differentiation. Scientifica (Cairo) 2013;2013:684736.

10 Fakhry M, Hamade E, Badran B, Buchet R, Magne D: Molecular mechanisms of mesenchymal stem cell differentiation towards osteoblasts. World J Stem Cells 2013;5:136-148.

11 Varelas X: The Hippo pathway effectors TAZ and YAP in development, homeostasis and disease. Development 2014;141:1614-1626.

12 Hao J, Zhang Y, Wang Y, Ye R, Qiu J, Zhao Z, Li J: Role of extracellular matrix and YAP/TAZ in cell fate determination. Cell Signal 2014;26:186-191.

13 Jeong GO, Shin SH, Seo EJ, Kwon YW, Heo SC, Kim KH, Yoon MS, Suh DS, Kim JH: TAZ mediates lysophosphatidic acid-induced migration and proliferation of epithelial ovarian cancer cells. Cell Physiol Biochem 2013;32:253-263.

14 Piccolo S, Dupont S, Cordenonsi M: The biology of YAP/TAZ: hippo signaling and beyond. Physiol Rev 2014;94:1287-1312.

15 Byun MR, Jeong H, Bae SJ, Kim AR, Hwang ES, Hong JH: TAZ is required for the osteogenic and antiadipogenic activities of kaempferol. Bone 2012;50:364-372.

16 Schreiweis MA, Butler JP, Kulkarni NH, Knierman MD, Higgs RE, Halladay DL, Onyia JE, Hale JE: A proteomic analysis of adult rat bone reveals the presence of cartilage/chondrocyte markers. J Cell Biochem 2007;101:466-476.

17 Gambacciani M, Levancini M: Hormone replacement therapy and the prevention of postmenopausal osteoporosis. Prz Menopauzalny 2014;13:213-220.

18 Body JJ: How to manage postmenopausal osteoporosis? Acta Clin Belg 2011;66:443-447.

19 Eriksen EF: Hormone replacement therapy or SERMS in the long term treatment of osteoporosis. Minerva Ginecol 2012;64:207-221.

20 Durão SF, Gomes PS, Colaço BJ, Silva JC, Fonseca HM, Duarte JR, Felino AC, Fernandes MH: The biomaterialmediated healing of critical size bone defects in the ovariectomized rat. Osteoporos Int 2014;25:15351545.

21 Gundberg CM: Biochemical markers of bone formation. Clin Lab Med 2000;20:489-501.

22 Gagnon C, Ebeling PR: Recent advances in managing osteoporosis. F1000 Med Rep DOI:10.3410/M1-96.

23 Kawai M, Mödder UI, Khosla S, Rosen CJ: Emerging therapeutic opportunities for skeletal restoration. Nat Rev Drug Discov 2011;10:141-156.

24 Guan F, Shi G, Zhao J, Wang Y: Progress on ovine lentivirus and its resistant genes. Yi Chuan 2014;36:12041210. 